\title{
STAY WITH CHESS
}

\section{E. Heinz $z^{1}$}

Karlsruhe, Germany

The single sentence below summarizes my personal opinion about the future of our organization and the ICCA Journal in particular:

"As mandated by its name, the ICCA should keep its focus on computer-chess while continuing to disseminate domain-dependent knowledge thereof and domain-independent knowledge from other fields relevant to computer-chess through its ICCA Journal."

Because there are already enough associations/organizations embracing specific computer games other than chess (e.g., Computer Shogi Association, CSA), I do not believe in aimless diversification of the ICCA towards unsolved, computerizable games. Instead, let us proceed with an ICCA dedicated to computer chess!

As for the notion of chess being solved, I violently disagree with Bob Herschberg's and Jaap van den Herik's editorial in the June 1996 ICCA Journal (Vol. 19, No. 2). The fact that more than $99 \%$ of all human chess-players regularly lose to today's state-of-the-art chess machines and programs - some of which are indeed able to produce acceptable commentary of their moves in certain types of positions - does not in itself establish anything even close to a solution of the game of chess. Of course, you may define any computerizable game to be "solved" as soon as the above condition holds, i.e., more than $99 \%$ of all human players regularly lose to state-of-the-art machines or programs exhibiting some degree of "understanding" when playing the game.

Obviously, this definition lacks the typical kind of scientific purity that most people intuitively combine with the solution of a game (see also related discussions in the newsgroup "rec.games.chess.computer" in June/July 1996). The general notion of a game being solved is that we can determine without doubt the final outcome or at least the best next move at any time during the course thereof. For chess, this certainly does not yet hold and it is questionable if it ever will!

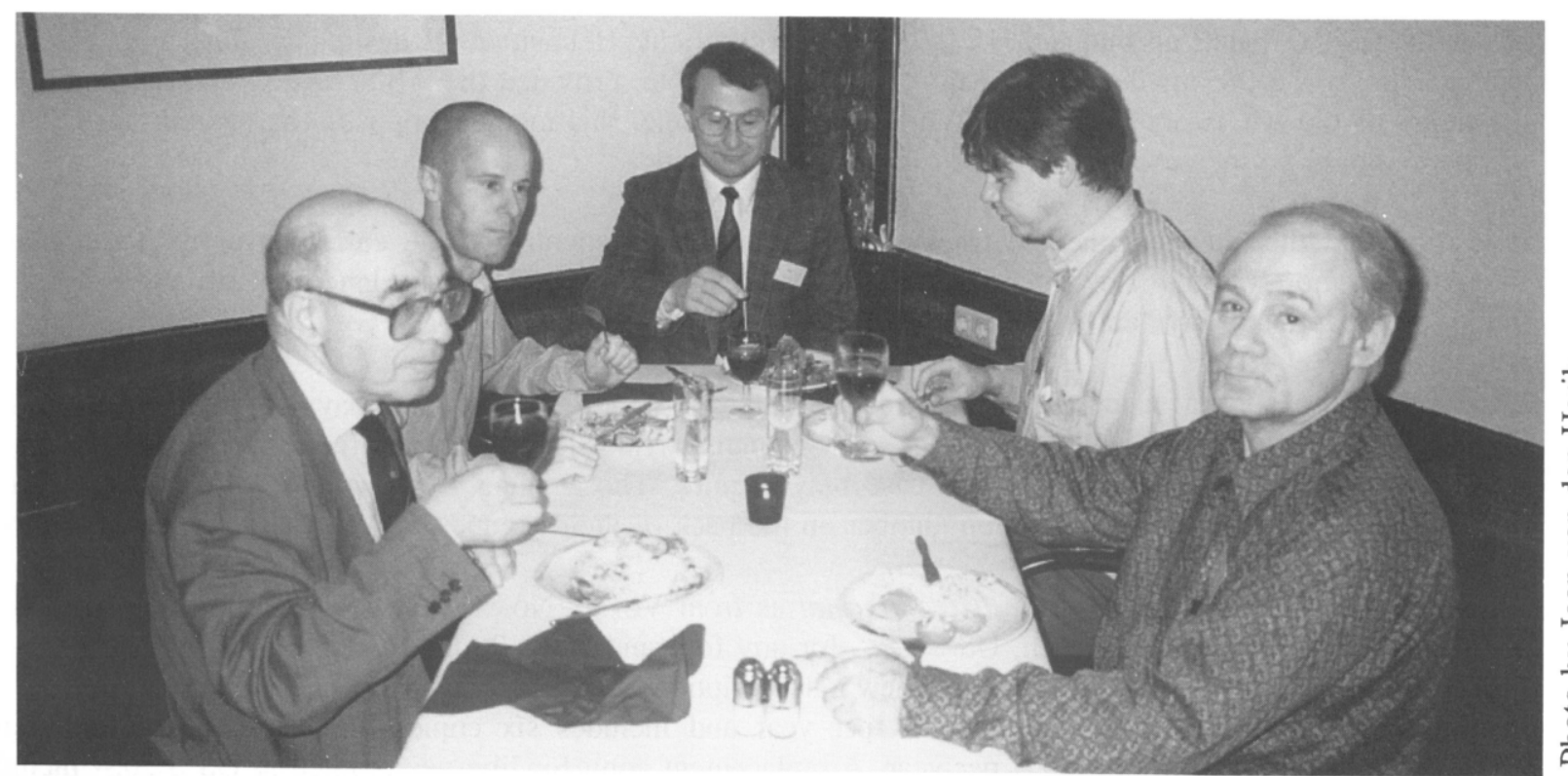

THE MIGHTY MEETING

Bronstein, the Guest of Honour, sitting opposite Tom Fürstenberg, his executive.

In the background, Frans Morsch, FRITZ's author, opposed to Johan de Koning, THE KING's author, at the far end Rainer Staudte from Chemnitz supervising them.

Vaeshartelt, Maastricht, June 27, 1996.

1 IPD School of Computer Science, University of Karlsruhe, P.O. Box 6980, D-76128 Karlsruhe, F.R. Germany. 